\title{
A BRIEF SURVEY OF SPHERICAL INTERPOLATION AND APPROXIMATION METHODS FOR TEXTURE ANALYSIS
}

\author{
H. SCHAEBEN \\ Guerickestr. 62, 66123 Saarbrücken, FRG
}

(Received 31 August 1995)

\begin{abstract}
In texture analysis there are several instances when mathematical methods of spherical interpolation or approximation are required. Ad hoc adaptions of univariate or bivariate methods to the topology of spherical manifolds usually fail in one way or another. Therefore, this contribution will provide a brief survey of genuinely spherical methods.
\end{abstract}

KEY WORDS: Spherical bilinear in terpolation, quasi-interpolation, spherical singular integrals, spherical multiquadrics, spherical splines.

\section{MOTIVATION AND INTRODUCTION}

The most prominent functions of texture analysis, the orientation density function describing the probability distribution of proper rotations, and the pole density function describing the probability distribution of crystallographic lattice planes are genuinely spherical functions. The orientation density function is defined on the threedimensional projective space equivalent to the upper (lower) unit sphere $H^{3} \equiv S_{+}^{4} \subset I R^{4}$, the pole density function is defined on the upper (lower) unit sphere $H^{2} \equiv S_{+}^{3} \subset I R^{3}$. Thus there are several instances when mathematical methods of spherical interpolation or approximation are required, e.g.

- preprocessing of experimental pole figure data,

- postprocessing of calculated values of a pole density function,

- display of an orientation density function,

- density estimation of individual orientation data;

Ad hoc adaptions of general multivariate methods (cf. Schumaker, 1976a, 1976b; Franke and Schumaker, 1987) to the topology of spherical manifolds usually fail in one way or another. They may be successful provided the domain of the data and of the interpolant/approximant to be constructed is a sufficiently small subset of $S^{d}$, or if it suffices that smoothness constraints or boundary conditions are only approximately satisfied for a particular subset of $S^{d}$, cf. Dierckx (1984); Gmelig Meyling and Pfluger (1987). However, if the domain of the interpolating or smoothing function to be constructed contains the north (south) pole of the sphere and if smoothness constraints or boundary conditions have to be exactly satisfied genuine spherical methods (cf. 
Schumaker and Traas, 1991; Traas et al., 1993) are required, because there is no differentiable mapping of the entire sphere to a bounded hyperplane. Therefore, this contribution will provide a brief survey of genuinely spherical methods. References of the topic include Berens et al., 1968; Pawelke, 1972; Butzer et al., 1979; Freeden, 1981; 1984; Wahba, 1981, 1984; Wehrens, 1981; Lawson, 1984; Renka, 1984; Barnhill et al., 1987; Nielson and Ramaraj, 1987; Foley, 1990; Pottmann and Eck, 1990; Schumaker and Traas, 1991; Hoschek and Seemann, 1992; Taijeron et al., 1994.

\section{BILINEAR SPHERICAL INTERPOLATION}

From the times of mechanical control of the texture goniometer two "equi-angular" grids to sample a pole density function have survived and are widely used. They are such that

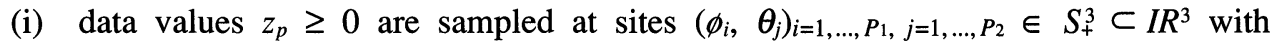
$\phi_{i}=-\pi+i \Delta \phi, i=0, \ldots, P_{1} ; P_{1}=2 \pi / \Delta \phi$ and $\theta_{j}=j \Delta \theta, j=0, \ldots, P_{2} ; P_{2}=\pi / \Delta \theta$;

(ii) data values $z_{p} \geq 0$ are sampled at sites $\phi_{i}=-\pi+\left(i-\frac{1}{2}\right) \Delta \phi, i=1, \ldots, P_{1}$ and $\theta_{j}=\left(j-\frac{1}{2}\right) \Delta \theta, j=1, \ldots, P_{2} \in S_{+}^{3}$.

Thus, the two grids are related to one another by a shift of half the angular step size in each spherical coordinate.

For a conversion of one equi-angular grid to the other bilinear spherical interpolation seems to provide the most simple and actually effective means. For $(\phi, \theta)$ in the patch defined by the four vertices $\left(\phi_{i p}, \theta_{j p}\right), p=1, \ldots, 4$, it reads

$$
\hat{z}(\phi, \theta)=\sum_{p=1}^{4} w_{p} z_{p}=\sum_{p=1}^{4} w_{p} z\left(\phi_{i p}, \theta_{j p}\right)
$$

where $w_{p}$ denotes the areal portion $f_{p} / F$ with

$$
f_{p}=\left|\phi-\phi_{i p}\right| \times\left|\cos (\theta)-\cos \left(\theta_{j p}\right)\right|
$$

of the total area $F=\sum_{p=1}^{4} f_{p}$ "diagonally across" the point $\left(\phi_{i p}, \theta_{j p}\right)$. Thus

$$
\hat{z}\left(\phi_{i}, \theta_{j}\right)=\mathrm{z}\left(\phi_{i}, \theta_{j}\right), i=1, \ldots, P_{1}, j=1, \ldots, P_{2}
$$

Particularly, for $\hat{z}(\phi, \pi / 2), \phi \in[0, \pi]$,

$$
\hat{z}(\phi, \pi / 2)=\hat{z}(\phi-\pi, \pi / 2)=\frac{z(\varnothing, \pi / 2-\Delta \theta / 2)+z(\varnothing-\pi, \pi / 2-\Delta \theta / 2)}{2}
$$

and for $\hat{z}(0,0)$

$$
\hat{z}(0,0)=\frac{1}{P_{1}} \sum_{p=1}^{P_{1}} z\left(\phi_{p}, \Delta \theta / 2\right)
$$

i.e. no extrapolation is needed.

Obviously, interpolated values are always nonnegative, and the resulting surface $\hat{z}$ is $C^{0}$. Repeated conversion of one grid to the other by bilinear interpolation results in an ever smoothed interpolant with gradually diminishing modes. Spherical bilinear interpolation does not seem appropriate for scattered data, i.e. for data sites arbitrarily distributed on the sphere. 


\section{APPROXIMATION BY SINGULAR CONVOLUTION INTEGRALS, QUASI-INTERPOLATION}

The basic idea (cf. Butzer and Nessel, 1971) is to approximate a (given) function $f$ by a function with "better" properties which is to be constructed by some smoothing operation on $f$ itself. The smoothing operation may be provided by convolution, which reads for spherical functions

$$
\left(f * \chi_{\rho}\right)(\mathbf{v})=\operatorname{area}^{-1}\left(S^{d}\right) \int_{S^{d}} f(\mathbf{u}) \chi_{\rho}(\mathbf{v u}) d s(\mathbf{u}), \mathbf{u}, \mathbf{v} \in S^{d}
$$

The convolution integral (3) is called a singlular integral (cf. Aronszajn, 1950) if the sequence $\left(\chi_{\rho}\right)_{\rho \in A}$ is a kernel, or since it is to be applied to probability density functions more specifically a nonnegative approximate identity. For a fixed $\mathbf{v}$ and a variable $\mathbf{u}$ of $S^{d} \subset I R^{d}$ the functions $\chi_{\rho}(\mathbf{v u})$ usually display a bell-shaped graph rotationally symmetric with respect to $\mathbf{v}$ with normalized area under the curve whereby for $\rho \rightarrow$ $\rho_{0}$ the mode at $\mathbf{v}$ becomes larger and narrower in such a way that the area under the curve near $\mathbf{v}$ comes out to be 1 . $A$ kernel with some parameter set $A$, or more precisely a nonnegative approximate identity $\left(\chi_{\rho}\right)_{\rho \in A}$ is defined by its properties

$$
\begin{gathered}
0 \leq \chi_{\rho}(t) \in L^{1}(-1,1), \quad \rho \in A \\
\frac{1}{2} \int_{(-1,1)} \chi_{\rho}(t) d t=1, \quad \rho \in A \\
\lim _{n \rightarrow \infty} \rho_{n}=\rho_{0} \\
\lim _{\rho_{n} \rightarrow \rho_{0}} \int_{\{-1 \leq t \leq 1-\delta\}} \chi_{\rho n}(t) d t=0,0<\delta<2 \\
\lim _{\rho_{n} \rightarrow \rho_{0}} \sup _{-1 \leq t \leq 1-\delta} \chi_{\rho n}(t)=0,0<\delta<2
\end{gathered}
$$

The last two properties may be used alternatively. The nonnegativity of the kernel implies its uniform boundedness with respect to the $L^{1}$ - norm.

The name approximate identity originates in the property that the sequence $\left\{f * \chi_{\rho}\right\}$ tends uniformly to $f$ as $\rho \rightarrow \rho_{0}$ which can be seen from

$$
\left(f * \chi_{\rho}\right)(\mathrm{v}) \approx f(\mathrm{v}) \operatorname{area}^{-1}\left(S^{d}\right) \int_{(1-\delta, 1)} \chi_{\rho}(t) d t
$$

One of the most prominent features of the convolution integral $f * \chi_{\rho}$ is that it preserves the "best" properties of each of its factors, i.e. if $\chi_{\rho}$ is very smooth, then $f * \chi_{\rho}$ will be so, too; and usually, $\chi_{\rho}$ are chosen to be very smooth.

Now it is presumed that the data values $z_{p} \geq 0, p=1, \ldots, P$, have been sampled from a spherical function $f \in \mathcal{L}^{q}\left(S^{3}\right)$ at arbitrarily scattered data sites $\mathbf{r}_{p}, p=1, \ldots$, $P$, i.e. $\left(\mathbf{r}_{p}, z_{p}\right)=\left(\mathbf{r}_{p}, f\left(\mathbf{r}_{p}\right)\right), p=1, \ldots, P$, provides the available data. Then an approximation of $f$ is given by

$$
\hat{f}\left(\mathbf{r} ; \rho_{P}\right)=\frac{1}{4 \pi} \sum_{p=1}^{P} f\left(\mathbf{r}_{p}\right) \chi_{\rho p}\left(\mathbf{r r}_{p}\right)=\frac{1}{4 \pi} \sum_{p=1}^{P} z_{p} \chi_{\rho p}\left(\cos \theta_{p}\right)
$$

with $\cos \theta_{p}=\mathbf{r r}_{p}$. 
It is emphasized that $\hat{f}\left(\mathbf{r} ; \rho_{P}\right)$ is nonnegative and a density itself of the same class of functions as the approximate identity $\chi_{\rho}$. The approximant $\hat{f}$ does not interpolate the data. In fact, it is given as superposition of rotationally invariant decreasing functions each of which centered at a data site $\mathbf{r}_{p}$ and multiplied by $z_{p}=f\left(\mathbf{r}_{p}\right)$. This approximation method may be referred to as "quasi-interpolation" because the interpolation conditions are thought of as being relaxed by the requirement that the dependence of $\hat{f}\left(\mathbf{r} ; \rho_{P}\right)$ with respect to each particular $f\left(\mathbf{r}_{p}\right)$ diminishes rapidly with increasing distance of $\mathbf{r}$ from $\mathbf{r}_{p}$.

Of major interest in practical applications with finite data sets $\left(\mathbf{r}_{p}, f\left(\mathbf{r}_{p}\right)\right), p=1, \ldots$, $P$, is not the sequence $f * \chi_{\rho}$ and its convergence but an individual member $\hat{f}=$ $f * \chi_{\rho_{p}}$ with a fixed parameter $\rho_{P}$ depending on the total number $P$ of data. This fixed parameter $\rho_{P}$ may be interpreted as the "window width" considering the data and controls the degree of smoothing. Therefore the choice of this tuning parameter is crucial. Several mathematical methods exist to optimize the choice of this parameter. Whatever the choice is, the degree of smoothing is usually large because one generally chooses "very smooth" functions as kernel, e.g. spherical analogues of the Gaussian probability density function (Nikolayev and Ullemeyer, these proceedings). Therefore, repeated application of these methods ("iterated singular integrals") tends to make characteristics of the data disappear.

While the parameter $\rho_{P}$ can be optimized for a given approximate identity, the choice of the approximate identity itself is not obvious. Early constructions of approximate identities which are optimal with respect to the rate of convergence are given by Bartlett (1963), Epachenikov (1969).

As an example for $S^{3} \subset I R^{3}$ without theoretical justification, the exponential function

$$
\chi_{\rho}(t)=c(\rho) \exp (\rho t) t \in(-1,1), \rho \in I R_{+}
$$

with

$$
\begin{aligned}
c(0) & =1 \\
c(\rho) & =\frac{\rho}{\sinh (\rho)} \\
\lim _{n \rightarrow \infty} \rho_{n} & =\rho_{0}=\infty
\end{aligned}
$$

borrowed from the zonal Langevin - von Mises - Fisher density is chosen as approximate identity. This choice yields the approximation

$$
\hat{f}(\mathbf{r} ; \rho p)=\frac{1}{4 \pi} \frac{\rho_{P}}{\sinh \rho_{P}} \sum_{p=1}^{P} f\left(\mathbf{r}_{p}\right) \exp \left(\rho_{P} \mathbf{r} \mathbf{r}_{p}\right)
$$

As another computationally less demanding example one may choose the de la Vallée Poussin approximate identity

$$
\chi_{n}(\cos \theta)=\frac{n !^{2}}{(2 n) !}\left(2 \cos \frac{\theta}{2}\right)^{2 n}, n \in I N
$$

The reader may find CosiPoWi (Adam, 1989) a rather peculiar reference to the de la Vallée Poussin kernel. 
Obviously, approximation methods by singular convolution integral generalize easily to hyperspheres of any dimension.

\section{INTERPOLATION BY RADIAL BASIS FUNCTIONS}

Employing radial basis functions for the purposes of interpolation/approximation or estimation seems to originate in the earth sciences, cf. Krige, 1951; Matheron, 1967; Crain and Bhattacharyya, 1967; Shepard, 1968. Radial interpolation methods are closely related to approximation by singular integrals (cf. Aronszajn, 1950; Butzer and Nessel, 1971) and splines as delivered by variational calculus (cf. Hogervorst, 1994). A recent survey is given by Kansa (1992).

The basic idea is to start with an analogue of (9), namely

$$
\hat{f}(\mathbf{r})=\sum_{p=1}^{P} \lambda_{p} \chi_{p}(\mathbf{r})=\sum_{p=1}^{P} \lambda_{p} \chi\left(\mathbf{r r}_{p}\right)=\sum_{p=1}^{P} \lambda_{p} \chi\left(\cos \theta_{p}\right)
$$

with $\cos \theta_{p}=\mathbf{r r}_{p}$ to construct a smooth function $\hat{f}$ such that (i) it interpolates, i.e.

$$
\hat{f}\left(\mathbf{r}_{p}\right)=f\left(\mathbf{r}_{p}\right)=z_{p}, p=1, \ldots, P
$$

is satisfied, (ii) $\chi_{p}$ depends only on the data site $\mathbf{r}_{p}$ and not on the surrounding data, and (iii) it is rotationally symmetric, or invariant, with respect to $\mathbf{r}_{p}$. The unknown coefficients $\lambda_{p}, p=1, \ldots, P$, shall be provided by eqs. (17).

Many schemes arising from this approach have the pleasent properties that (i) interpolation is always possible and unique under very mild conditions on the spatial arrangement of data sites, (ii) the interpolant matches the required smoothness, and (iii) they generalize easily to higher dimensions or non-Euclidean manifolds as hyperspheres.

Examples of radial functions $\chi: I R+\mapsto I R$ frequently applied in multi-variate Euclidean interpolation are (cf. Hogervorst, 1994)

$$
\begin{aligned}
\text { surface } & \chi(r)=r^{2 n-1}, n \in I N \\
\text { thin plate } & \chi(r)=r^{2 n} \log r, n \in I N \\
\text { multiquadric } & \chi(r)=\left(r^{2}+c^{2}\right)^{1 / 2}, c \in I R \\
\text { inverse multiquadric } & \chi(r)=\left(r^{2}+c^{2}\right)^{-1 / 2}, c \in I R \\
\text { shifted surface } & \chi(r)=\left(r^{2}+c^{2}\right)^{n-1 / 2}, n \in I N, c \in I R \\
\text { shifted thin plate } & \chi(r)=\left(r^{2}+c^{2}\right)^{n} \log \left(r^{2}+c^{2}\right), n \in I N, c \in I R \\
\text { shifted logarithm } & \chi(r)=\log \left(r^{2}+c^{2}\right), c \in I R \\
\text { Gaussian } & \chi(r)=\exp \left(-c r^{2}\right), c \in I R_{+}
\end{aligned}
$$

where $\chi_{p}(\mathrm{x})=\chi\left(r_{p}\right)$ with $r_{p}=\left\|\mathrm{x}-\mathrm{x}_{p}\right\|, \mathrm{x}, \mathrm{x}_{p} \in I R^{d}, d \in I N$. 
The surface and thin plate radial basis function can be derived from variational calculus (cf. Hogervorst, 1994). The multiquadric as well as the inverse multiquadric has been introduced by Hardy (1971, 1990), Hardy and Goepfert (1975), Hardy and Nelson (1986) in geophysics. Shifted relatives have been introduced by Dyn et al. (1986), Dyn (1989). The Gaussian reveals most obviously the close relation to approximation by the singular integral of Gauss-Weierstrass. All of them may be read as appropriately smoothed multivariate versions of univariate piecewise linear interpolation provided by $\chi_{\rho}(x)=$ $\chi\left(\left|x-x_{p}\right|\right)$ which produces discontinuities of the first derivative at the data sites $x_{p}$. In case of the multiquadrics smoothing of the distance function is done by using arcs of hyperbolas that possess the $r_{p}$ values as asymptotes, where $c$ is the tuning parameter which must be supplied by the user. Considering the augmented data sites $\left(\mathrm{x}_{p}, 0\right)$ and $\left(\mathrm{x}_{p}, c\right)$ as elements of $I R^{d+1}$, the multiquadric $\chi_{\rho}(\mathrm{x})$ may be interpreted as the distance between these augmented data sites.

Next, generalizations of the multiquadric and inverse multiquadric approach for scattered spherical data are summarized as they are known for their excellent practical achievements (Foley, 1990; Pottmann and Eck, 1990).

Let $g_{p}(\mathbf{r})=\arccos \left(\mathbf{r r}_{p}\right)$ denote the geodesic distance of $\mathbf{r}$ from $\mathbf{r}_{p}$. Then define

$$
\hat{f}(\mathbf{r})=\sum_{p=1}^{P} \lambda_{p} \chi\left(g_{p}(\mathbf{r})\right)
$$

where $\chi(t)$ is either the multiquadric or the inverse multiquadric. As usually, coefficients $\lambda_{p}, p=1, \ldots, P$, are determined by solving the linear system of equations

$$
\hat{f}\left(\mathbf{r}_{p}\right)=\sum_{p=1}^{P} c_{j} B\left(g_{j}\left(\mathrm{r}_{p}\right)\right)=z_{p}, p=1, \ldots, P
$$

Each basis function $\chi\left(g_{p}(\mathbf{r})\right)$ is a $C^{\infty}$ function on the sphere except at the antipodal point $-\mathbf{r}_{p}$, where it is not differentiable. Thus the interpolant is $C^{0}\left(S^{d}\right)$.

Modifications of basis functions such that they are $C^{2}$ on $S^{d}$ can be accomplished by piecewise quintic poynomial blending near the antipodal point.

(i) Modifying the geodesic distance function

$$
\hat{f}(\mathbf{r})=\sum_{p=1}^{P} \lambda_{p} \chi\left(h_{p}(\mathbf{r})\right)
$$

where

$$
h_{p}(\mathbf{r})=\left\{\begin{array}{l}
g_{p}(\mathbf{r}) \text { if } g_{p}(\mathbf{r}) \leq 3 \\
H\left(g_{p}(\mathbf{r})\right) \text { otherwise }
\end{array}\right.
$$

and $H(t)$ is the polynomial of degree $\leq 5$ that satisfies $H(3)=3, H^{\prime}(3)=1, H^{\prime \prime}(3)$ $=0$ and $H(\pi)=3.1, H^{\prime}(\pi)=0, H^{\prime \prime}(\pi)=0 ; H(t)$ is convex and monotone on $[3, \pi]$. Then $\chi\left(h_{p}(\mathbf{r})\right), \hat{f}(\mathbf{r})$ are $C^{2}$ in $S^{d}$.

(ii) Modifying the basis function itself

$$
\hat{f}(\mathbf{r})=\sum_{p=1}^{P} \lambda_{p} \psi\left(g_{p}(\mathbf{r})\right)
$$


where

$$
\psi(t)=\left\{\begin{array}{l}
\chi(t) \text { if } t \leq 3 \\
\Psi(t) \text { if } 3<\mathrm{t} \leq \pi
\end{array}\right.
$$

and $\Psi(t)$ is the polynomial of degree $\leq 5$ that satisfies $\Psi(3)=\chi(3), \Psi(\pi)=\chi(3.1)$, $\Psi^{\prime}(3)=\chi^{\prime}(3), \Psi^{\prime \prime}(\pi)=0, \Psi^{\prime \prime}(3)=\chi^{\prime \prime}(3), \Psi^{\prime \prime}(\pi)=0$; then $\psi\left(g_{p}(\mathbf{r})\right)$ is $C^{2}$ on $S^{d}$, hence $\hat{f}(\mathbf{r})$ is so, too.

In practical applications the constant $c^{2}$ has to be chosen. Empirically, a value of $c^{2}=2.2$ area $\left(S^{d}\right) / P$ yielded consistently effective results.

Yet another spherical generalization (Hardy and Goepfert, 1975; Pottmann and Eck, 1990) is defined as

$$
\hat{f}(\mathbf{r})=\sum_{p=1}^{P} \lambda_{p} \chi_{p}(\mathbf{r})
$$

where

$$
\chi_{p}(\mathbf{r})=\sqrt{1+c^{2}-2 c\left(\mathbf{r r}_{p}\right)}
$$

usually with $0<c<1$, but $c>1$ is feasible, too. $c=3 / 8$ yields $\chi_{p}\left(\mathbf{r}_{p}\right)=5 / 8$; for large $P$ a smaller value of $c$ is to be preferred; an optimal choice of $c$ is unknown.

The linear system of equations to determine the coefficients has always a unique solution (Micchelli, 1986). However, the system may be ill conditioned, in particular for large $P$, and require preconditioning (cf. Hogervorst, 1994). The interpolant is not necessarily nonnegative. If the nonnegativity constraint is actually violated for one tuning parameter $c_{1}$ another value $c_{2}$ may apply.

\section{APPROXIMATION BY SPHERICAL SPLINES}

Polynomial splines may roughly be thought of as piecewise polynomials of a given polynomial order which are joined together to form a unique function at their defining knots. With polynomial interpolation/approximation in mind one may think of the decoupling of the total number of interpolation conditions (data) and the polynomial order of the interpolant as one major motivation of the development of polynomial splines. In accordance with the best known natural cubic splies the term spline is nowadays used for a function that is defined piecewise or satisfies a specific optimality constraint or both. Key references for splines in probability and statistics are provided by Boneva et al., 1971; Wegman and Wright, 1983; Silverman, 1989.

This communication is confined to an approximation scheme provided by a tensor product of a polynomial and a periodic trigonometric spline of order $m=n=3$ (Schumaker and Traas, 1991; Traas et al., 1993).

It is again presumed that the data values $z_{p} \geq 0, p=1, \ldots, P$, have been sampled from a spherical function $f \in \mathcal{L}^{q}\left(S^{3}\right)$ at arbitrarily scattered data sites $\mathbf{r}_{p}, p=1, \ldots$, $P$, i.e. $\left(\mathbf{r}_{p}, z_{p}\right)=\left(\mathbf{r}_{p}, f\left(\mathbf{r}_{p}\right)\right), p=1, \ldots, P$, provides the available data. Let the function $f(\mathbf{r})=f(\vartheta, \varphi)$ be given in terms of spherical coordinates lattitude $\vartheta \in[0, \pi]$ and longitude $\varphi \in[0,2 \pi]$ of $\mathbf{r} \in S^{3}$.

Assume the spline approximation $\hat{f}(\mathbf{r})$ of $f(\mathbf{r})$ is given as

$$
\hat{f}(\mathbf{r})=\hat{f}(\vartheta, \varphi)=\sum_{i=1}^{M} \sum_{j=1}^{N+2} c_{i j} N_{i}^{m}(\vartheta) T_{j}^{n}(\varphi)
$$


where $N_{i}^{m}, m=1, \ldots, M$, are the usual normalized B-splines of order $m$ associated with the extended partition

$$
x_{1}=\ldots=x_{m}=-\frac{\pi}{2}<x_{m+1}<\ldots<x_{M}<\frac{\pi}{2}=x_{M+1}=\ldots=x_{M+m}
$$

and where $T_{j}^{n}, n=1, \ldots, N$, are the normalized periodic trigonometric B-splines of order $n$ associated with the partition

$$
\begin{aligned}
& 0=y_{n}<y_{n+1}<\ldots<y_{N+n-1}<y_{N+n}=2 \pi \\
& y_{i}=y_{i+N}-2 \pi \text { and } y_{N+n+i}=y_{n+i}+2 \pi, i=1, \ldots, n-1
\end{aligned}
$$

Desirable properties of a well defined $\hat{f}(\vartheta, \varphi)$ are

- data fit in the sense of

$$
\hat{f}\left(\mathbf{r}_{p}\right) \approx f\left(\mathbf{r}_{p}\right), p=1, \ldots, P
$$

- continuity

$$
\begin{array}{r}
f(\vartheta, 0)=f(\vartheta, 2 \pi),-\pi / 2 \leq \vartheta \leq \pi / 2 \\
f(-\pi / 2, \varphi)=f_{\mathcal{S}} \text { and } f(\pi / 2, \varphi)=f_{N}, 0 \leq \varphi \leq 2 \pi
\end{array}
$$

- continuously varying tangent plane

$$
\begin{gathered}
\frac{\partial}{\partial \varphi} f(\vartheta, 0)=\frac{\partial}{\partial \varphi} f(\vartheta, 2 \pi),-\pi / 2 \leq \vartheta \leq \pi / 2 \\
\frac{\partial}{\partial \vartheta} f(-\pi / 2, \varphi)=A_{s} \cos \varphi+B_{s} \sin \varphi, 0 \leq \varphi \leq 2 \pi \\
\frac{\partial}{\partial \vartheta} f(\pi / 2, \varphi)=A_{N} \cos \varphi+B_{N} \sin \varphi, 0 \leq \varphi \leq 2 \pi
\end{gathered}
$$

where $A_{\mathcal{S}}, B_{\mathcal{S}}, A_{N}, B_{N}$ are constants.

These side conditions can be written in the form

$$
B \mathbf{c}=0
$$

Data is fit by minimizing the mean square error

$$
E(\mathbf{c})=\sum_{p=1}^{P}\left[\hat{f}\left(\mathbf{r}_{p}\right)-f\left(\mathbf{r}_{p}\right)\right]^{2} \rightarrow \min
$$

Summarily, that is to find the best $l^{2}$ solution of the system of equations

$$
\sum_{i=1}^{M} \sum_{j=1}^{N+2} c_{i j} N_{i}^{m}\left(\vartheta_{p}\right) T_{j}^{n}\left(\varphi_{p}\right)=f\left(\mathbf{r}_{\mathrm{p}}\right), p=1, \ldots, P
$$


or equivalently

subject to

$$
A \mathbf{c}=\left(f\left(\mathbf{r}_{1}\right), \ldots, f\left(\mathbf{r}_{p}\right)\right)^{t}
$$

$$
B \mathbf{c}=0
$$

A solution can be obtained be the Householder transformation method. The approximate is $C^{1}$ on $S^{3}$.

This scheme of spline approximation generalizes to a better order of smoothness as well as to hyperspheres in a straightforward manner. Its major advantage is that repeated application does not change the initial spline approximation, because the spline approximation of a given spline is this spline itself. While B-splines are nonnegative functions any linear combination need not to be nonnegative. If the required nonnegativity of $\hat{f}$ is violated it may be corrected by appropriate subdivision (cf. Dyn et al., 1990) and final correction of corresponding control points or some other numerical heuristics. Promising results have been reported by Traas et al. (1993).

\section{CONCLUSIONS}

This communication is merely a brief review of methods of genuinely spherical interpolation and approximation. It is in no way complete, but rather a subjective selection of suggested readings. Any major omission is owed to the author's limited knowledge.

\section{References}

Adam, J. F. (1989). Methoden und Algorithmen zur Verwaltung und Analyse axialer 3-D-Richtungsdaten und ihrer Belegungsdichten: Diss. Univ. Göttingen, Göttinger Arb. Geol. Paläont. 40, Göttingen.

Aronszajn, N. (1950). Theory of reproducing kernels: Trans. Amer. Math. Soc., 68, 337-404.

Barnhill, R. E., Piper, B. R. and Rescorla, K. L. (1987). Interpolation to arbitrary data on a surface: in Farin, G., (ed.), Geometric Modeling, SIAM, Philadelphia, PA, 281-289.

Bartlett, M. S. (1963). Statistical estimation of density functions: Sankhya A 25, 245-254.

Berens, H., Butzer, P. L. and Pawelke, S. (1968). Limitierungsverfahren von Reihen mehrdimensionaler Kugelfunktionen und deren Saturationsverhalten: Publ. RIMS, Kyoto Univ., Ser. A, 4, 201-268.

Boneva, L. I., Kendall, D. and Stefanov, I. (1971). Spline transformations: Three new diagnostic aids for the statistical data-analyst: J. Roy. Statist. Soc., ser. B, 33, 1-71.

Butser, P. L. and Nessel, R. J. (1971). Fourier Analysis and Approximation: Birkhäuser Verlag, Basel and Stuttgart.

Butzer, P. L., Stens, R. L. and Wehrens, H. (1979). Approximation by algebraic convolution integrals: in Prolla, J. B., (ed.), Approximation Theory and Functional Analysis, North Holland Publishing Comp, 71-120.

Crain, I. K. and Bhattacharyya, B. K. (1967). Treatment of non-equispaced two-dimensional data with a digital computer: Geoexploration, 5, 173-194.

Dierckx, P. (1984). Algorithms for smoothing data on the sphere with tensor product splines: Computing, 32, 319-342.

Dyn, N. (1989). Interpolation and approximation by radial and related functions: in Chui, C. K., Schumaker, L. L. and Ward, J. D., (eds.), Approximation Theory VI, Vol., 1, Academic Press, 211-234.

Dyn, N., Levin, D. and Liu, D. (1990). Interpolatory convexity preserving subdivision schemes for curves and surfaces: preprint.

Dyn, N., Levin, D. and Rippa, S. (1986). Numerical procedures for surface fitting of scattered data by radial functions: SIAM J. Sci. Comput., 7, 639-659.

Epachenikov, V. A. (1969). Nonparametric estimates of a multivariate probability density: Theor. Prob. Appl., 14, 153-158. 
Foley, T. A. (1990). Interpolation to scattered data on a spherical domain: in Mason, J. C. and Cox, M. G., (eds.), Algorithms for Approximation II, Chapman and Hall, London, 303-310.

Franke, R. and Schumaker, L. L. (1987). A bibliography of multivariate approximation: in Chui, C. K., Schumaker, L. L. and Utreras, F. I., Topics in Multivariate Approximation, Academic Press, 275-335.

Freeden, W. (1981). On spherical spline interpolation and approximation: Math. Methods Appl. Sci., 3, 551-575.

Freeden, W. (1984). Spherical spline interpolation: Basic theory and computational aspects: J. Comput. App. Math., 11, 367-375.

Freeden, W. and Hermann, P. (1986). Uniform approximation by spherical spline approximation: Math. Z., 193, 265-275.

Gmelig Meyling, R. H. J. and Pfluger, P. R. (1987). B-spline approximation of a closed surface: IMA J. Numer. Anal., 7, 73-96.

Hardy, R. L. (1971). Multiquadric equations of topography and other irregular surfaces: J. Geophys. Res., 76, 1905-1925.

Hardy, R. L. (1990). Theory and application of the multiquadric-biharmonic method: Comput. Math. Applic., 19, 163-208.

Hardy, R. L. and Goepfert, W. M. (1975). Least squares predictions of gravity anomalies, geoidal undulations, and deflections of the vertical with multiquadric harmonic functions: Geophys. Res. Lett., 2, 423-426.

Hardy, R. L. and Nelson, S. A. (1986). A multiquadric biharmonic representation and approximation of disturbing potential: Geophys. Res. Lett., 13, 18-21.

Hogervorst, B. J. (1994). Developments in spline interpolation: PhD Thesis, Enschede, The Netherlands

Hoschek, J. and Seemann, G. (1992). Spherical splines: Mathematical Modelling and Numerical Analysis 26, 1-22.

Kansa, E. J., ed., (1992). Advances in the theory and applications of radial basis functions: Computers Math. Applic., 24(12).

Krige, D. G. (1951). A statistical approach to some basic mine valuation problems on the Witwatersrand: $\mathrm{PhD}$ thesis, University of the Witwatersrand.

Lawson, C. L. (1984). $C^{1}$ surface interpolation for scattered data on a sphere: Rocky Mountain J. Math., 14, 177-202.

Matheron, G. (1967). Kriging or polynomial interpolation: Transactions of the Canadian Institute of Mining and Metallurgy, 70, 240-244.

Micchelli, C. A. (1986). Interpolation of scattered data: distance matrices and conditionally positive definite functions: Constr. Approx., 2, 11-22.

Nielson, G. M. and Ramaraj, R., 1987, Interpolation over a sphere based upon a minimum norm network: $C A G D, 4,41-57$.

Nikolayev, D. and Ullemeyer, K. (1995). Texture data processing: the effect of smoothing on odf reproduction: these proceedings.

Pawelke, S. (1972). Über die Approximationsordnung bei Kugelfunktionen und algebraischen Polynomen: Tohoku Math. Journ., 24, 473-486.

Pottmann, H. and Eck, M. (1990). Modified multiquadric methods for scattered data interpolation over a sphere: $C A G D, 7,313-321$.

Renka, R. J. (1984). Interpolation of data on the surface of a sphere: ACM Trans. Math. Software, 10, $417-436$.

Schumaker, L. L. (1976a). Two-stage spline methods for fitting surfaces: in Schaback, R. and Scherer, K., (eds.), Approximation Theory, Springer, 378-389.

Schumaker, L. L. (1976b). Fitting surfaces to scattered data: in Lorentz, G.G. and Schumaker, L.L., Approximation Theory II, Academic Press, 203-268.

Schumaker, L. L. and Traas, C. R. (1991). Fitting scattered data on spherelike surfaces using tensor products of trigonometric and polynomial splines: Numer. Math., 60, 133-144.

Shepard, D. (1968). A two dimensional interpolation function for irregularly spaced data: Proc 23rd Nat. Conf. ACM, 517-524.

Silverman, B. W. (1989). Spline smoothing: the equivalent variable kernel method: Ann. Statist., 12, 898-916.

Taijeron, H. J., Gibson, A. G. and Chandler, C. (1994). Spline interpolation and smoothing on hyperspheres: SIAM J. Sci. Comput., 15, 1111-1125.

Traas, C. R., Siemes, H. and Schaeben, H. (1993). Smoothing pole figures using tensor products of trigonometric and polynomial splines: Materials Science Forum 157-162 (Proceedings ICOTOM 10), $453-458$. 
Wahba, G. (1981). Spline interpolation and smoothing on the sphere: SIAM J. Sci. Stat. Comput., 2, 5-16; Erratum: SIAM J. Sci. Stat. Comput., 3, 385-386.

Wahba, G. (1984). Surface fitting with scattered noisy data on Euclidean d-space and on the sphere: Rocky Mountain J. Math., 14, 281-299.

Wegman, E. J. and Wright, I. W. (1983). Splines in statistics: J. Amer. Stat. Assoc., 78, 351-365.

Wehrens, M. (1981). Approximationstheorie auf der Einheitskugel im $I R^{3}$. Legendre Transformationsmethoden und Anwendungen: Westdeutscher Verlag, Opladen. 